\title{
Comments on the paper by Horowitz et al. (2014)
}

\author{
G. M. Innocenti $\cdot$ R. Caminiti $\cdot$ F. Aboitiz
}

Published online: 13 January 2015

(c) Springer-Verlag Berlin Heidelberg 2015

We have been impressed by the previous work of Assaf and collaborators, in particular that reporting differences of axon diameter in different sectors of the corpus callosum with applications of water diffusion methods (Barazany et al. 2009). That work returned the differences that have been well-documented histologically in macaque, chimpanzee, and humans by several groups including ourselves. Using histological as well as diffusion tractography (DT), two of us have unequivocally demonstrated that axon diameter differences in the corpus callosum and elsewhere relate both to the area of origin and to the termination of the projections. We have also computed the conduction delays that axon diameters and tract lengths generate in the brain and the predictions fit well the available, albeit scarce, electrophysiological evidence (Caminiti et al. 2009; Tomasi et al. 2012; Innocenti et al. 2013; Caminiti et al. 2013).

This comment refers to the article available at doi:10.1007/s00429-014-0871-0.

G. M. Innocenti $(\bowtie)$

Department of Neuroscience, Karolinska Institutet, Retzius Väg 8, 17177 Stockholm, Sweden

e-mail: giorgio.innocenti@ki.se

G. M. Innocenti

Brain and Mind Institute, EPFL, 1015 Lausanne, Switzerland

R. Caminiti

Department of Physiology and Pharmacology,

University of Rome Sapienza, 00185 Rome, Italy

F. Aboitiz

Department of Psychiatry Medical School, Interdisicplinary Center for Neuroscience, Pontificia Universidad Católica de Chile, 8330024 Santiago, Chile
We are less impressed with the results reported by Horowitz et al. in Brain Struct Funct 2014, for the following reasons:

1. The diameter of callosal axons in the splenium estimated from DT $(3.5 \mu \mathrm{m})$ is considerably larger than that measured histologically by light microscopy

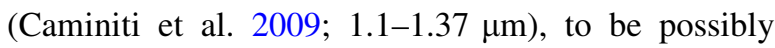
corrected to $1.5-1.8 \mu \mathrm{m}$ by the usually accepted shrinkage factor of $30 \%$. Contrary to what Horowitz et al. claim, it is also far from the EM measurements of Aboitiz et al. (1992; in the order of 1 um in their Fig. 4), to be possibly corrected to $1.3 \mu \mathrm{m}$ due to shrinkage. Fibers larger than $3 \mu \mathrm{m}$ made no more than $1 \%$ of the total fibers. Notice that the EM might have returned slightly smaller axons than the light microscopy since the latter could have marginally underestimated the proportion of small axons.

2. The interhemispheric transfer times computed from visually evoked responses $(4.8 \mathrm{~ms})$ are much shorter than those estimated by 4 other groups $(16-20 \mathrm{~ms}$, quoted in Tomasi et al. 2012; see also Aboitiz et al. 1992, 2003). The paper by Whitford et al. (2011) similarly reports interhemispheric transfer times above $10 \mathrm{~ms}$, albeit with individual variabilities. These interhemispheric delays are compatible with those calculated from light microscopic measurements of callosal axon diameters and lengths histologically and with DT in humans (Caminiti et al. 2013).

3. The interhemispheric transfer time computed from somatosensory stimuli $(3.9 \mathrm{~ms})$ is also shorter than what can be found in the literature. Moreover, it is claimed that it was recorded from the postcentral gyrus (SI) where due to our knowledge bilateral responses were not reported in humans, but inhibitory interaction 
were, at 20-25 ms delays (Ragert et al. 2011). Instead bilateral somatosensory responses were recorded from S2 with latencies, in the order of 12.4 (Frot and Mauguière 1999) and 17.4 (Stancak et al. 2002).

4. Horowitz et al. believe that they recorded visual interhemispheric transfer time generated by axons interconnecting areas 17 and 18 of the two hemispheres. Unfortunately, these axons are very few since it is common knowledge that they interconnect only the midline of the visual hemifields (near the vertical meridian). Moreover, these axons run in a narrow, horizontal sector of the ventral splenium as it was well documented in both monkey and in humans histologically and with DT (Caminiti et al. 2013). These axons would be very difficult to identify with DT. It is therefore likely that the evoked responses they recorded and the axons they measured were between the peristriate areas.

5. Even if, as we suggest, the visual interhemispheric transfer time was due to the activation of peristriate connections, it is definitely too short, and it cannot be excluded that it may be due to scattered light activating directly (but with low intensity) the hemifield contralateral to the stimulation.

It seems obvious that the DT estimate of axon diameter is biased toward large axons. In other words, DT does not yet resolve axons in the 1-2 $\mu \mathrm{m}$ range, which constitute the majority of cortical connections, although several laboratories are trying to get there. This is not to be blamed but the paper would have been improved had this been properly stated.

Our advice is that the authors correct their paper, in order not to damage the excellent reputation acquired this far.

It is nevertheless satisfactory to see that in spite of the limitations of their methods, the authors still report shorter interhemispheric delays between somatosensory than between visual areas in line with the work of two of us, quoted above.

\section{References}

Aboitiz F, Scheibel AB, Fisher RS, Zaidel E (1992) Fiber composition of the human corpus callosum. Brain Res 598:143-153

AboitizF López J, Montiel J (2003) Long distance communication in the human brain: timing constraints for interhemispheric synchrony and the origin of brain lateralization. Biol Res 36:89-99

Barazany D, Basser PJ, Assaf Y (2009) In vivo measurement of axon diameter distribution in the corpus callosum of rat brain. Brain 132:1210-1220

Caminiti R, Ghaziri H, Galuske R, Hof PR, Innocenti GM (2009) Evolution amplified processing with temporally dispersed slow neuronal connectivity in primates. Proc Natl Acad Sci USA 106:19551-19556

Caminiti R, Carducci F, Piervincenzi C, Confalone G, BattagliaMayer A, Visco-Comandini F, Patrizia Pantano P, Innocenti GM (2013) Diameter, length, speed and conduction delay of callosal axons in macaque monkeys and humans: comparing histology and DTI data. J Neurosci 33:14501-14511

Frot M, Mauguière F (1999) Timing and spatial distribution of somatosensory responses recorded in the upper bank of the sylvian fissure (SII area) in humans. Cereb Cortex 9:851-863

Innocenti GM, Vercelli A, Caminiti R (2013) The diameter of cortical axons depends both on area of origin and termination. Cereb Cortex 24:2178-8218

Ragert P, Nierhaus T, Cohen LG, Villringer A (2011) Interhemispheric interactions between the human primary somatosensory cortices. PLoS One 6:e16150

Stancak A, Hoechstetter K, Tintera J, Vrana J, Rachmanova R et al (2002) Source activity in the human secondary somatosensory cortex depends on the size of corpus callosum. Brain Res 936:47-57

Tomasi S, Caminiti R, Innocenti GM (2012) Areal differences in diameter and length of corticofugal projections. Cereb Cortex 22:1463-1472

Whitford TJ, Kubicki M, Ghorashi S, Schneiderman JS, Hawley KJ, McCarley RW, Shenton ME, Spencer KM (2011) Predicting inter-hemispheric transfer time from the diffusion properties of the corpus callosum in healthy individuals and schizophrenia patients: a combined ERP and DTI study. Neuroimage $54: 2318-2329$ 